\title{
Implementação, Avaliação e Comparação dos Protocolos de Desmame com Tubo-T e Pressão Suporte Associada à Pressão Expiratória Final Positiva em Pacientes Submetidos à Ventilação Mecânica por mais de 48 Horas em Unidade de Terapia Intensiva*
}

\author{
Implementation, Assessment and Comparison of the T-Tube and \\ Pressure-Support Weaning Protocols Applied to the Intensive Care Unit \\ Patients who had Received Mechanical Ventilation for more than 48 Hours
}

Tatiane Colombo', Aline Fiorillo Boldrini², Silvia Renata Rezek Juliano ${ }^{3}$, Maria Cecilia Rezek Juliano4, João Geraldo Simões Houly5, Otavio Celso Eluf Gebara ${ }^{6}$, Gil Vicente L. Cividanes ${ }^{7}$, Elaine C. Catão8.

\section{RESUMO}

JUSTIFICATIVA E OBJETIVOS: A retirada precoce da ventilação mecânica dos pacientes das unidades de terapia intensiva (UTI) é importante para a redução da morbimortalidade, porém na prática, os desmames são

1. Fisioterapeuta dos Hospitais Santa Paula e São Camilo e Aprimoranda em Fisioterapia Cardiorrespiratória pelo InCor HC-FMUSP.

2. Fisioterapeuta dos Hospitais Santa Paula e São Luiz, Especialista em Fisiologia do Exercício na Saúde na Doença e no Envelhecimento.

3. Fisioterapeuta Coordenadora do Serviço de Fisioterapia e Especializada em Fisioterapia Cardiorrespiratória pelo InCor HC FMUSP.

4. Fisioterapeuta Coordenadora do Serviço de Fisioterapia do Hospital Santa Paula.

5. Médico Coordenador da Unidade de Terapia Intensiva Adulto do Hospital Santa Paula.

6. Médico Coordenador da Unidade Coronariana do Hospital Santa Paula.

7. Médico Coordenador da Unidade de Terapia Intensiva B do Hospital Santa Paula.

8. Psicóloga Doutora em Psicologia Escolar e Desenvolvimento Humano - IPUSP

${ }^{*}$ Recebido da Unidade de Terapia Intensiva do Hospital Santa Paula, São Paulo, SP

Apresentado em 09 de dezembro de 2006.

Aceito para publicação em 12 de fevereiro de 2007

Endereço para correspondência:

Serviço de Fisioterapia - Hospital Santa Paula

Avenida Santo Amaro ${ }^{\circ} 2468$

04556-100 - $3^{\circ}$ Andar Ala B - São Paulo, SP

Fone: (11) 30408166

E-mail: fisioterapia@santapaula.com.br

(C)Associação de Medicina Intensiva Brasileira, 2007 realizados aleatoriamente. Face à importância desse procedimento, esse estudo avaliou a implementação de protocolos de desmame e comparou dois métodos distintos.

MÉTODO: Foram incluídos no estudo 120 pacientes dependentes de ventilação mecânica por mais de 48 horas. O método de Pressão Suporte + PEEP (PSP), foi aplicado aos pacientes em dias pares, constituindo o grupo 1 (GPSP) e em dias ímpares, utilizou-se o método do Tubo-T (TT), formando o grupo 2 (GTT),

RESULTADOS: A resposta dos pacientes à extubação revelou evolução semelhante nos dois grupos, porém deixou claro, pela análise estatística do teste Qui-quadrado, o benefício de se utilizar um protocolo de desmame. De todos os pacientes estudados, 109 (90,83\%) tiveram sucesso na extubação não sendo necessário nenhum tipo de ventilação não-invasiva dentro de 24 horas após o desmame, enquanto que apenas 11 pacientes $(9,17 \%)$ necessitaram de ventilação mecânica não-invasiva ou de re-intubação no mesmo período, caracterizando o insucesso do desmame.

CONCLUSÕES: A implementação e a padronização de protocolos de desmame da ventilação mecânica, reduziu significativamente o índice de re-intubação na UTI, diminuindo o período de internação e o índice de morbimortalidade, porém neste estudo, não foram encontradas diferenças estatísticas significativas entre os métodos analisados.

Unitermos: desmame, implantação, método Tubo-T, protocolo de ventilação mecânica, ventilação com pressão suporte 


\section{SUMMARY}

BACKGROUND AND OBJECTIVES: Mechanical ventilation incurs significant morbidity and mortality, weaning intensive care unit patients is highly desirable, although it is usually conducted in an empirical manner. Thus, this article assessed a weaning protocol implementation and compared two different methods.

METHODS: It was carried out a study involving 120 patients who had received mechanical ventilation for more than 48 hours. These patients were randomly assigned to undergo one of two weaning techniques: pressuresupport ventilation + PEEP (PSP) technique, which was applied to the patients in equal days, forming the PSP group (PSPG) and the T-tube method (TT), applied in odd days and forming the TT group (TTG). Standardized protocols were followed for each technique

RESULTS The patients response to extubation revealed similar progress in both PSP and $T$ groups, but after the Chi-square statistical test, the benefits of using a weaning protocol was clear. One hundred nine $(90.83 \%)$ of all patients, had a successful weaning and any noninvasive ventilation type was needed in a span time of 24 hours after extubation, and only eleven (9.17\%), had an unsuccessful weaning.

CONCLUSIONS: Although this study didn't show any difference between the two methods applied, we could conclude that, the implementation of standardized weaning protocols can substantially decrease the patient's reintubation rate, promoting a downward trend in mortality and morbidity for these patients and shortening their hospital and intensive care units length of stay.

Key Words: implementation, mechanical ventilation protocol, pressure-support ventilation, T-tube method, weaning

\section{INTRODUÇÃO}

O desmame é definido como um processo de transição da ventilação mecânica para a ventilação espontânea'. O processo de desmame pode ser dividido em três fases, isto é, desmame durante a ventilação, extubação e desmame do oxigênio suplementar. A primeira fase é freqüentemente iniciada com a diminuição dos parâmetros do ventilador, gradativamente, até o paciente conseguir total independência ventilatória. A fase seguinte consiste na retirada da prótese ventilatória, sendo oferecido, caso necessário, oxigênio suplementar. $E$ por fim, o desmame gradual do oxigênio suplementar.
Muitos pacientes são extubados diretamente no modo Pressão Suporte + PEEP, enquanto outros poderão ser extubados através do tubo- ${ }^{2}$.

Segundo o II Consenso Brasileiro de Ventilação Mecânica ${ }^{3}$, aproximadamente $60 \%$ a $70 \%$ dos pacientes criticamente enfermos e ventilados artificialmente podem ser extubados após breve teste de duas horas de ventilação espontânea ${ }^{3}$.

Segundo a literatura, além do momento adequado para seu início, a escolha do método de desmame também influenciam no sucesso ou insucesso da extubação, bem como na morbimortalidade dos pacientes extubados ${ }^{4}$. Inúmeros estudos sobre desmame tem sido publicados nos últimos anos, refletindo o interesse em se reduzir o tempo total de ventilação mecânica e apontar os melhores métodos de liberação do paciente do ventilador. Porem, os estudos até então publicados, não concluem de forma clara sobre qual o método mais eficiente.

A padronização de um protocolo de desmame em UTI, pode atender melhor os requisitos de eficiência e segurança do procedimento, favorecendo a evolução e diminuindo a incidência de complicações.

O objetivo deste estudo foi implantar um protocolo de desmame da ventilação mecânica e comparar dois métodos distintos.

\section{MÉTODO}

Após aprovação pelo Comitê de Ética do Hospital Santa Paula, foi realizado um estudo aleatório e prospectivo pela equipe Médica e da Fisioterapia. O estudo foi aprovado pela equipe multidisciplinar do hospital. Participaram do estudo 120 pacientes internados nas UTI A e B e Unidade Coronariana que estavam sob ventilação mecânica prolongada (período maior que 48 horas). A coleta dos dados ocorreu no período de dezembro de 2004 a maio de 2006.

Foram incluídos todos os pacientes aptos ao desmame, independentemente do tipo de doença de base ou do evento que tenha motivado a ventilação mecânica. Foram excluídos do estudo os pacientes traqueostomizados ou que fizeram uso da prótese ventilatória por período menor que 48 horas. A escolha dos pacientes para composição da amostra ocorreu de maneira aleatória.

Os dados foram posteriormente tabulados, analisados e apresentados em tabelas e figuras. Para a análise estatística utilizou-se o teste do Qui-quadrado (homogeneidade para a comparação intra- 
IMPLEMENTAÇÃO, AVALIAÇÃO E COMPARAÇÃO DOS PROTOCOLOS DE DESMAME COM TUBO-T

E PRESSÃO SUPORTE ASSOCIADA À PRESSÃO EXPIRATÓRIA FINAL POSITIVA EM PACIENTES

SUBMETIDOS À VENTILAÇÃO MECÂNICA POR MAIS DE 48 HORAS EM UNIDADE DE TERAPIA INTENSIVA

grupo e independência para a comparação entre grupos), sendo considerado o nível de significância de 0,05 .

\section{Protocolo de Desmame}

O protocolo foi elaborado e aplicado pela equipe de fisioterapia envolvendo as seguintes fases:

- Pré-desmame: que consiste na avaliação do paciente a partir dos critérios clínicos e indicadores fisiológicos, a fim de se tomar a decisão de iniciar o processo de desmame;

- Desmame: que compreende a fase de interrupção da ventilação mecânica e a monitorização do paciente durante este período de autonomia ventilatória;

- Extubação: que é a retirada da prótese ventilatória, após duas horas de autonomia, permanecendo estável durante este período. Fazendo o uso de oxigênio suplementar após a retirada da ventilação superficial, sendo o paciente reavaliado a partir da gasometria coIhida 30 min após a extubação.

\section{Pré-Desmame}

Os pacientes que apresentaram melhoras ou resoluções da causa determinante da ventilação mecânica, foram analisados de maneira sistemática e diária pela equipe multidisciplinar, quanto aos critérios clínicos essenciais para indicação do processo de desmame, que correspondem aos critérios de inclusão no protocolo:

- Tempo de ventilação mecânica > 48 horas;

- Resolução ou controle da causa que levou a intubação traqueal;

- Suspensão ou diminuição a níveis mínimos de sedativos, bloqueadores neuromusculares e fármacos vasoativos;

- Presença de drive respiratório;

- Ausência de sepse ou estado gerador de hipertermia;

- Estabilidade hemodinâmica;

- Ausência de desordens metabólicas/eletrolíticas;

- Correção da sobrecarga hídrica;

- Nenhuma expectativa de procedimento cirúrgico de grande porte;

- Idealmente três horas de jejum ou esvaziamento gástrico via sonda;

- Teste do balonete vazio negativo (ausência de edema de glote);

- Parâmetros gasométricos $\mathrm{PaO}_{2}>60$ mmHg com
$\mathrm{FiO}_{2} \leq 40 \%$; $\mathrm{SatO}_{2}>90 \% ; \mathrm{pH}>7,30<7,60$ e $\mathrm{PaO}_{2} / \mathrm{FiO}_{2}$ $\geq 200$, obtidos através de gasometria arterial;

- Parâmetros ventilatórios PEEP $=5 \mathrm{cmH}_{2} \mathrm{O} ; \mathrm{PS}=7$ $\mathrm{cmH}_{2} \mathrm{O}$ e f $=0$.

- Foram considerados critérios de exclusão após aleatorização;

- Desenvolvimento de instabilidade hemodinâmica após o inicio do protocolo;

- Impossibilidade de atingir os parâmetros de mecânica ventilatória mínimos necessários (estabelecidos previamente);

- Extrema agitação ou ansiedade, impossibilitando o teste de ventilação espontânea por duas horas.

Os pacientes incluídos nos critérios clínicos, também deveriam satisfazer aos indicadores fisiológicos preditivos de sucesso no desmame por avaliarem melhor quanto a fadiga muscular, serem facilmente reprodutíveis e utilizarem instrumentos e técnicas simples e disponíveis ${ }^{3,5,6}$. São eles:

- $\mathrm{V}_{\mathrm{T}} 5$ a $8 \mathrm{~mL} / \mathrm{kg}$;

- $F R \leq 35$

- $P i m a x \leq-20 \mathrm{mmHg}$

- Índice de respiração rápida superficial < 100 ipm/L Os pacientes que satisfizeram as condições exigidas na etapa anterior, foram considerados aptos a efetuar a segunda etapa.

\section{Desmame}

Nesta fase do protocolo os pacientes foram submetidos ao teste de autonomia, com duração de duas horas em ventilação espontânea ${ }^{2}$. Ao serem considerados aptos ao desmame, os pacientes foram divididos aleatoriamente em dois grupos, que utilizaram métodos distintos - Tubo-T e PSV + PEEP (PEEP $=5+$ PS $=7)^{7}$. O procedimento de aleatorização adotado foi em relação ao dia do desmame. Em dias pares foram extubados a partir do modo Pressão Suporte associado à PEEP (G1) e dias impares Tubo-T (G2).

No $\mathrm{G} 1$, o desmame foi realizado até que os valores descritos fossem atingidos, não necessitando da desconexão da ventilação artificial, permanecendo por duas horas com esses parâmetros, de forma clinicamente estável.

No G2, o paciente foi desconectado do ventilador e em seu tubo traqueal conectou-se uma peça tipo $\mathrm{T}$, junto à uma fonte umidificada e enriquecida de oxigênio (5 L/ min), permanecendo também por duas horas de forma estável clinicamente (Tabela 1). 
Tabela 1 - Grupos Aleatórios do Protocolo

\begin{tabular}{ll}
\hline \multicolumn{1}{c}{$\mathrm{G} 1$} & \multicolumn{1}{c}{$\mathrm{G} 2$} \\
\hline Dias pares & Dias impares \\
CPAP (PS + PEEP) & Tubo- $\mathrm{T}$ \\
$\mathrm{PS}=7 \mathrm{cmH}_{2} \mathrm{O}$ & $\mathrm{FiO}_{2}=5 \mathrm{~L} / \mathrm{min}$ \\
$\mathrm{PEEP}=5 \mathrm{cmH}_{2} \mathrm{O}$ & $\mathrm{F}=0$ irpm \\
Duração: 2 horas & Duração: 2 horas \\
\hline
\end{tabular}

CPAP = pressão positiva contínua em vias aéreas; $\mathrm{PEEP}=$ pressão expiratória final positiva $P S=$ pressão de suporte

Durante o teste de autonomia, foi realizado monitorização continua dos parâmetros que correspondem aos sinais de intolerância à desconexão da ventilação mecânica (Tabela 2).

Tabela 2 - Sinais de Intolerância à Desconexão da Ventilação Mecânica $^{3}$

\begin{tabular}{lc}
\hline Parâmetros & Intolerância \\
\hline Freqüência respiratória $_{\text {SatO }_{2}}$ & $>35$ irpm \\
Freqüência cardíaca & $<90 \%$ \\
Pressão arterial sistólica & $>140 \mathrm{bpm}$ \\
Sinais e sintomas & $<90>180 \mathrm{mmHg}$ \\
& Agitação, sudorese, alteração do \\
& nível de consciência \\
\hline
\end{tabular}

Os pacientes que apresentaram sinais de intolerância ao desmame, durante o período de duas horas de ventilação espontânea, retornaram às condições ventilatórias prévias ao desmame, sendo considerada falha do processo de desmame.

Ao final de duas horas, os pacientes que não apresentaram sinais de intolerância passavam para a próxima fase do protocolo.

\section{Extubação Traqueal}

Se necessário, dependendo do quadro ventilatório indicado pela ausculta pulmonar, foi realizado previamente à extubação traqueal, aspiração da cânula traqueal e das vias aérea superiores.

Procedeu-se em seguida o teste do balonete vazio, com o objetivo de prevenir complicações após a extubação. Se negativo, foi efetuada a extubação. Após a extubação era ofertado oxigênio suplementar, com máscara de nebulização (5 L/min), e em casos especiais cateter tipo óculos a $3 \mathrm{~L} / \mathrm{min}$. Após $30 \mathrm{~min}$ da extubação foi colhida gasometria arterial para análise dos dados gasométricos. Foram observados durante 24 horas, período indicado pelo Consenso, permanecendo em autonomia ventilatória e estabilidade clínica, sendo considerados como sucesso do desmame.

Em caso de necessidade de retorno ao ventilador, num período de $24 \mathrm{~h}$ foi considerado insucesso do desmame, sendo então adotada a estratégia de desmame gradual, adequada ao quadro clinico do paciente, ou um plano de reabilitação envolvendo treinamento muscular respiratório, caso o motivo do insucesso do desmame tenha mostrado ser fraqueza muscular ventilatória (Pinsp < 20).

A necessidade de ventilação não-invasiva devido ao quadro de desconforto respiratório (dispnéia, uso de musculatura acessória, sinais de fadiga muscular, dessaturação, taquipnéia e taquicardia) durante o pós-extubação também foi considerado falha do protocolo (Figura 1).

\section{RESULTADOS}

O estudo foi composto por 120 pacientes, de ambos os sexos (70 do sexo masculino e 50 do sexo feminino), com idades variadas, englobando diversas doenças de base e submetidos à ventilação mecânica por período maior que 48 horas.

A figura 2 apresenta a distribuição da população estudada de acordo com a faixa etária.

A tabela 3 apresenta as doenças de base mais observadas no período de avaliação sobre a implantação do protocolo, sua porcentagem em relação à amostra e a quantidade de pacientes que tiveram sucesso ou insucesso após a sua aplicação.

Nos casos pneumológicos as doenças mais freqüentes foram: tromboembolismo pulmonar, broncopneumonia, tuberculose pulmonar, doença pulmonar obstrutiva crônica (DPOC), pneumonia aspirativa.

Nos casos cardíacos: insuficiência cardíaca congestiva, infarto agudo do miocárdio, choque cardiogênico. Nos casos neurológicos: acidente vascular encefálico (hemorrágico / isquêmico), aneurisma cerebral.

Nos casos oncológicos: neoplasia de mama, neoplasia de reto, neoplasia de pâncreas e tumor cerebral.

Para os outros casos: úlcera gástrica, infecção do trato urinário, pós-operatório do trato gastrintestinal, neurológicos, cardíacos, ortopédicos, vasculares, sepse, insuficiência renal crônica, diabete melito, politraumatismo e grande queimado.

A figura 3 apresenta os dados de sucesso e insucesso de acordo com os métodos de desmame utilizados. Pode-se observar que em 120 pacientes estudados, obteve-se 109 casos de sucesso e apenas 11 de insucesso do desmame. O cálculo do Qui-quadrado de homogeneidade foi de 80,03 , para um grau de liberdade e significância de 0,00\% (significativo ao nível de 5\%), 
IMPLEMENTAÇÃO, AVALIAÇÃO E COMPARAÇÃO DOS PROTOCOLOS DE DESMAME COM TUBO-T

E PRESSÃO SUPORTE ASSOCIADA À PRESSÃO EXPIRATÓRIA FINAL POSITIVA EM PACIENTES

SUBMETIDOS À VENTILAÇÃO MECÂNICA POR MAIS DE 48 HORAS EM UNIDADE DE TERAPIA INTENSIVA

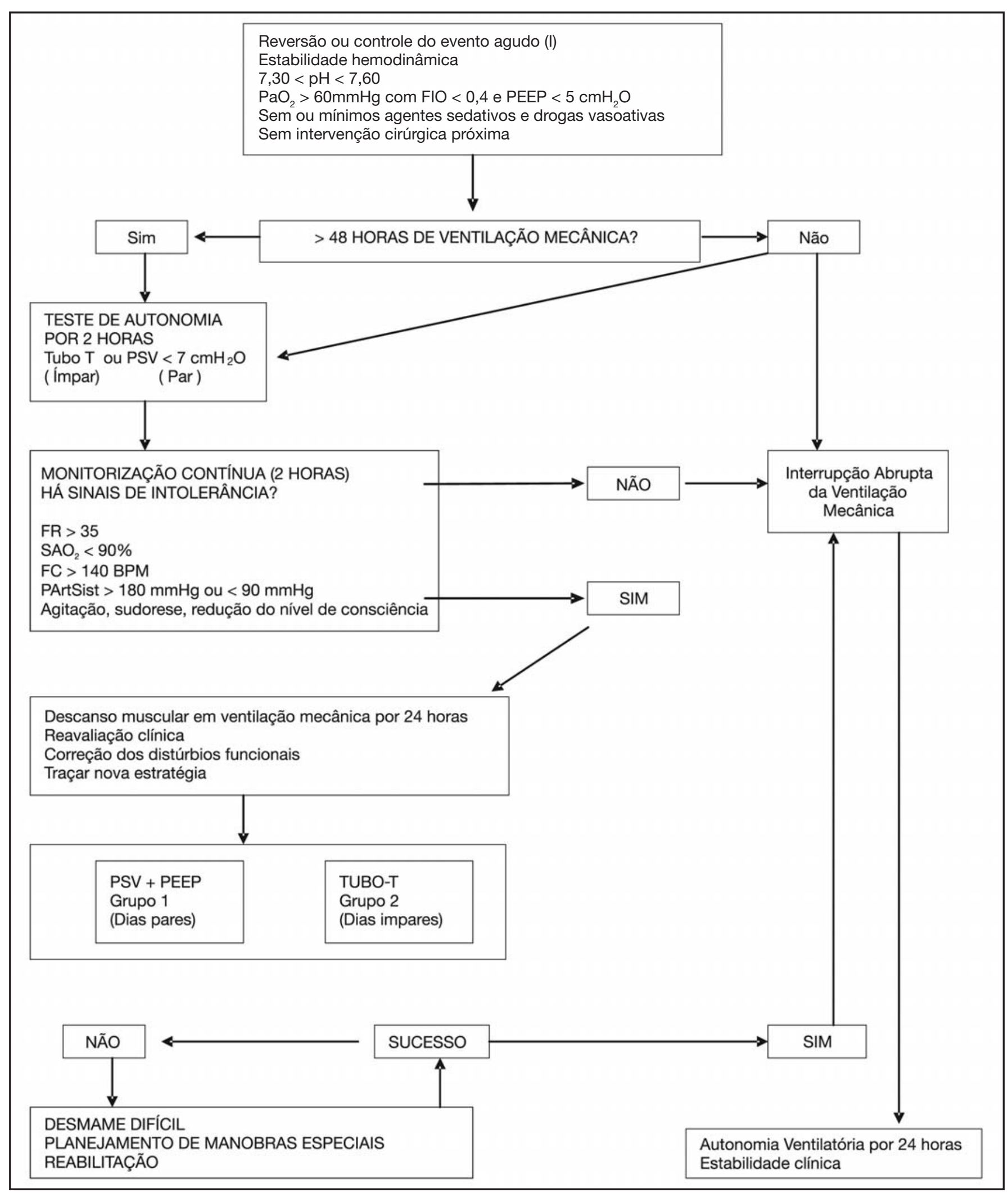

Figura 1 - Fluxograma do Protocolo de Desmame Baseado no II Consenso de Ventilação Mecânica. 


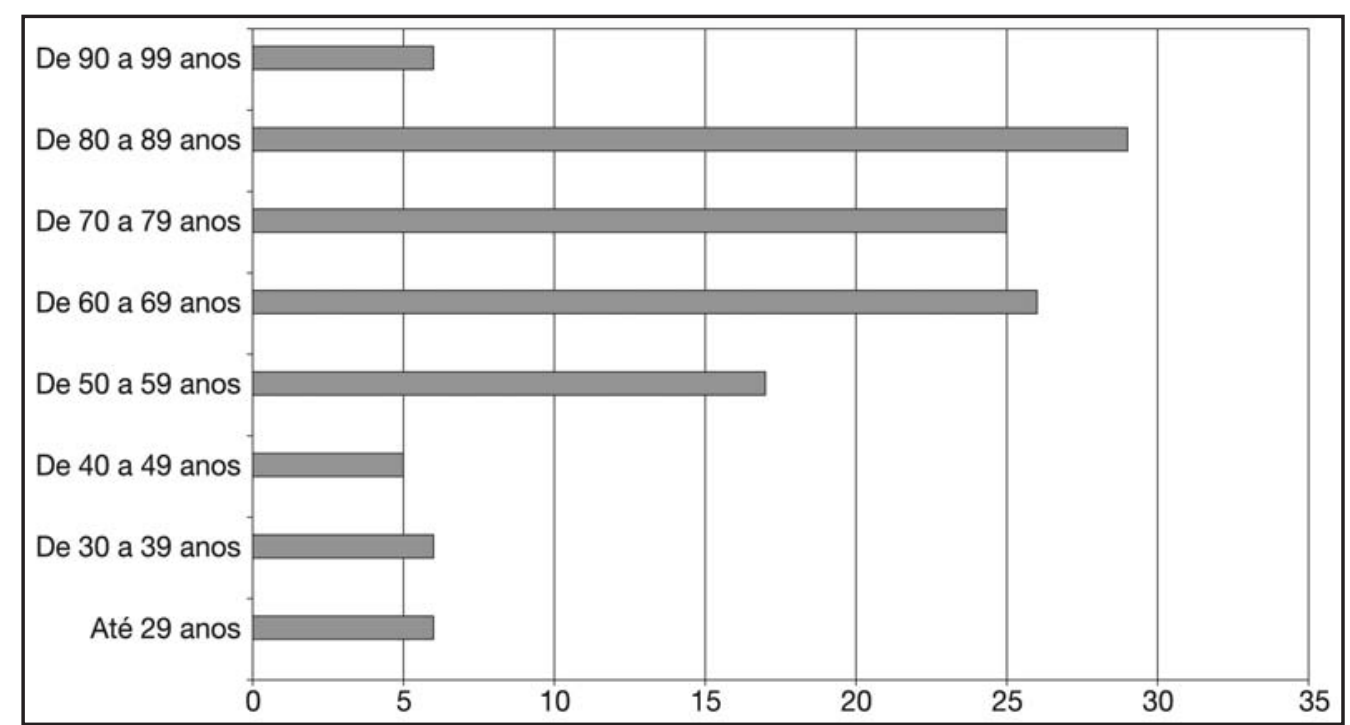

Figura 2 - Distribuição da População Estudada de acordo com a Faixa Etária

Tabela 3 - Doenças de Base, Métodos Utilizados e Índices de Sucesso e Insucesso de Desmame dos Pacientes Estudados

\begin{tabular}{|c|c|c|c|c|c|}
\hline Doenças & Grupos & $\mathrm{N}^{\circ}$ de Pacientes & $\%$ & Sucesso & Insucesso \\
\hline \multirow[t]{2}{*}{ Pneumológica } & G1 & 31 & 26 & 30 & 1 \\
\hline & G2 & 21 & 18 & 16 & 5 \\
\hline \multirow[t]{2}{*}{ Cardíaca } & G1 & 4 & 3 & 4 & - \\
\hline & G2 & 5 & 4 & 5 & - \\
\hline \multirow[t]{2}{*}{ Neurológica } & G1 & 18 & 15 & 16 & 2 \\
\hline & G2 & 6 & 5 & 4 & 2 \\
\hline \multirow[t]{2}{*}{ Oncológica } & G1 & 4 & 3 & 3 & 1 \\
\hline & G2 & - & - & - & - \\
\hline \multirow[t]{2}{*}{ Outra } & G1 & 17 & 14 & 17 & - \\
\hline & G2 & 14 & 12 & 14 & - \\
\hline
\end{tabular}

$\mathrm{G} 1=\mathrm{PS}+\mathrm{PEEP}$

$\mathrm{G} 2=$ Tubo- $\mathrm{T}$

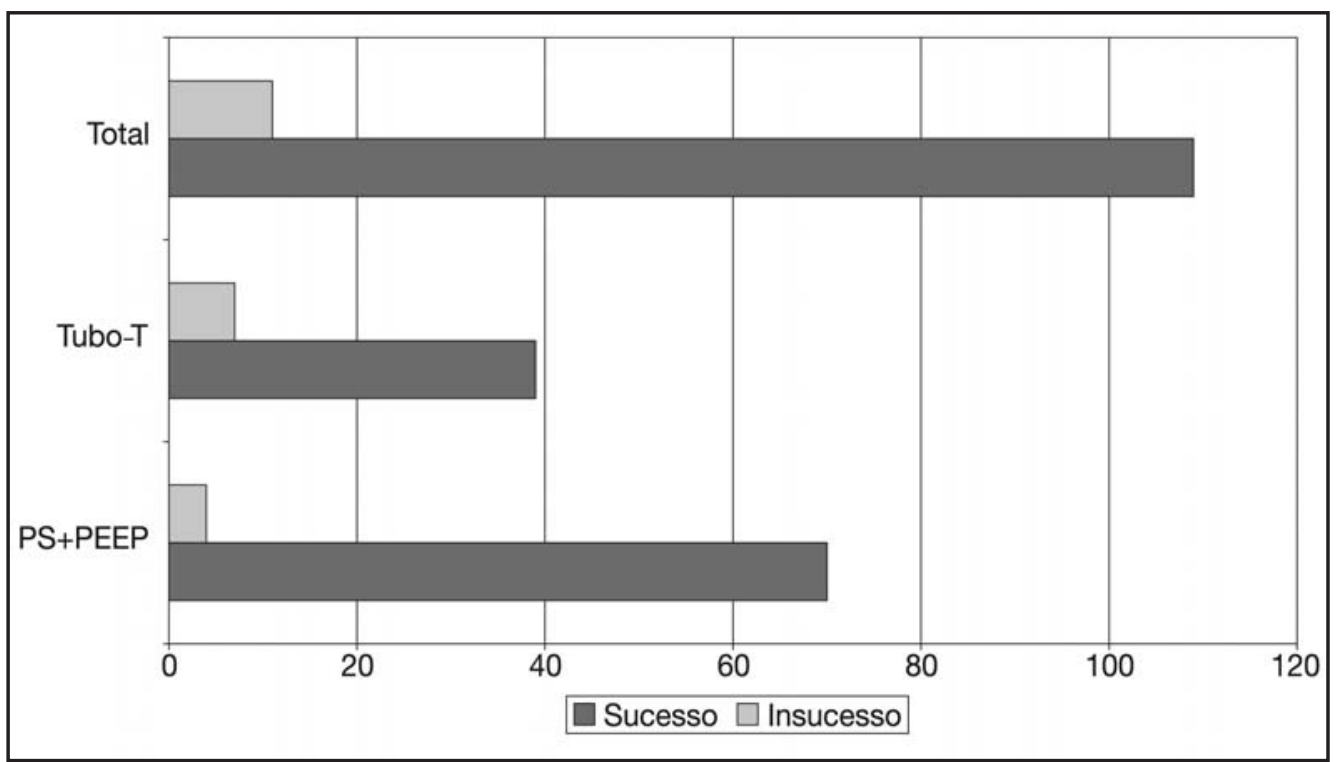

Figura 3 - Distribuição dos Casos de Sucesso e Insucesso no Processo de Desmame de acordo com o Tipo de Método Utilizado 
comprovando a eficácia da utilização do protocolo de desmame na UTI.

Quanto à comparação entre os tipos de métodos de desmame utilizados (PS+PEEP/G1 e Tubo-T/G2), o cálculo do Qui-quadrado de independência não evidenciou diferença significativa entre os G1 e G2 $\chi^{2}$ independência $=3,28$; graus de liberdade $=1$; significância $=$ 7,01\%; não significativo ao nível de 5\%), demonstrando, portanto, que tais métodos apresentaram níveis similares de sucesso e insucesso em sua utilização.

\section{DISCUSSÃO E CONCLUSÃO}

A partir dos dados levantados por meio do estudo em questão, pode-se observar que independente da escoIha do método de desmame da ventilação mecânica, da idade, do sexo ou da doença de base, faz-se necessário o estabelecimento de um protocolo de desmame para que se possa garantir maior índice de sucesso e menor incidência de comorbidades nos pacientes internados em UTI e dependentes da ventilação mecânica ${ }^{3,5-7,13}$.

Ao término de 17 meses de pesquisa, em que foram estudados 120 pacientes, concluiu-se que entre os métodos Tubo-T e PSV associada à PEEP, não houve diferença significativa ao nível de $0,05 \%$. Portanto, o processo de desmame deve ser priorizado, pois a manutenção do paciente em ventilação invasiva, coloca-o em risco de diversos eventos associados ao aumento da morbimortalidade $e^{2,8,10}$.

A partir dos resultados descritos foram observados que ao ser estabelecido um protocolo de desmame obteve-se diminuição do tempo de ventilação mecânica, da permanência do paciente em tal processo e, em conseqüência, do custo total de internação em UTI e, até mesmo do índice de mortalidade ${ }^{11-14}$.

A experiência prática aqui descrita ainda levou os au- tores a constatar uma melhora no atendimento aos pacientes deste estudo e uma interação mais adequada entre a equipe multidisciplinar.

\section{REFERÊNCIAS}

01. HessD-Ventilatormodesused inweaning. Chest,2001;120:(Suppl6):474S$476 S$.

02. Jones DP, Byrne P, Morgan C et al - Positive end-expiratory pressure vs T-piece. Extubation after mechanical ventilation. Chest, 1991;100:16551659.

03. II Consenso Brasileiro de Ventilação Mecânica - Desmame e Interrupção da Ventilação Mecânica. Ed. Atheneu, 2000.

04. Eliy EW - Weaning from mechanical ventilation: acute and chronic management. Chest, 2001;15:522S-529S

05. Meade M, Guyatt G, Cook D et al - Predicting success in weaning mechanical ventilation. Chest, 2001;120:(Suppl6):400S-424S.

06. Coberlline C, Trevisan CBE, Costa AD et al - Avaliação dos critérios convencionais preditivos de desmame de suporte ventilatório mecânico em pacientes idosos durante a ventilação espontânea com tubo-T. RBTI, 2003;15:2:58-63.

07. Carvalho CRR - Ventilação Mecânica Vol. I - Básico. São Paulo, Ed. Atheneu, 2001.

08. Meade M, Guyatt G, Sinuff T et al - Trials comparing alternative weaning modes and discontinuation assessments. Chest, 2001;120: (Suppl6):425S-437S

09. Tobin $\mathrm{MJ}$ - Advances in mechanical ventilation $\mathrm{N}$ Engl $\mathrm{J}$ Med, 2001;344:1896-1994.

10. Assunção MSC, Machado FR, Rosseti HB et al - Avaliação de teste de tubo-T como estratégia inicial de suspensão da ventilação mecânica. RBTI, 2006;18:121-125.

11. Krishnan JA, Moore D, Robeson C et al - A prospective, controlled trial of a protocol-based strategy to discontinue mechanical ventilation. Am J Respir Crit Care Med, 2004;169:673-678.

12. Esteban A, Alia I, Gordo F et al - Extubation outcome after spontaneous breathing trials with T-tube or pressure support ventilation. Am J Respir Crit Care Med, 1997; 156: 459-465.

13. Ely EW, Meade MO, Haponik EF et al - Mechanical ventilator weaning protocols driven by nonphysician health-care professionals: evidence based clinical practice guidelines. Chest, 2001;120:(Suppl6):454S-463S.

14. Oliveira LRC, Jose A, Dias ECP et al - Padronização do desmame da ventilação mecânica em unidade de terapia intensiva: resultados após um ano. RBTI, 2006;18:131-136.

15. Esteban A, Alia I, Tobin MJ et al - Effect of spontaneous breathing trial duration on outcome of attempts to discontinue mechanical ventilation. Am J Respir Crit Care Med, 1999;159:512-518.

16. Esteban A, Frutos F, Tobin MJ et al - A comparison of four methods of weaning patients from mechanical ventilation. N Engl J Med, 1995;332:345-350.

17. Brochard L, Rauss A, Benito $S$ et al - Comparison of three methods of gradual withdrawal from ventilatory support during weaning from mechanical ventilation. Am J Respir Crit Care Med, 1994; 150:896-903. 